\title{
Proposta de um método de otimização baseado em tendência para predição do comportamento de séries financeiras
}

\author{
Marcus V. C. Bastos* Eduardo G. Carrano** \\ Lucas S. Batista ${ }^{* *}$ \\ * Operations Research and Complex Systems Laboratory, Programa de \\ Pós-Graduação em Engenharia Elétrica, Universidade Federal de \\ Minas Gerais, MG, (e-mail: mvcbastos@gmail.com). \\ ** Operations Research and Complex Systems Laboratory, \\ Departamento de Engenharia Elétrica, Universidade Federal de Minas \\ Gerais, MG, (e-mails: egcarrano@ufmg.br, lusoba@ufmg.br)
}

\begin{abstract}
This paper deals with the forecasting problem of assets' prices in financial series from BM\&F Bovespa, the Brazilian stock exchange. The goal is to propose a forecasting method based on Artificial Neural Networks (ANNs) that focuses on adjusting the network weights in order to maximize the rate of correctness of the price trends of a given asset, instead of minimizing the error between its real values and predicted ones. At the same time, the method minimizes the probability of overfitting, which is a very frequent problem in ANN applications. Due to the characteristics of the problem found in its modeling stage, such as the presence of two objectives which one of them presents a discontinuous and non-differentiable function, a multiobjective evolutionary algorithm has been implemented to solve it. This work used real data from 10 assets and an index from BM\&F Bovespa. The computational experiments carried out indicate that the proposed model, although simple, presented competitive results when compared to the best works in the field, which use error minimization in its implementation. In this way, the presented approach was very promising, being able to contribute with more works of this line of research.

Resumo: Este trabalho aborda o problema de predição de preços de ativos em séries financeiras da BM\&F Bovespa, a bolsa de valores brasileira. O objetivo é propor um método de predição baseado em Redes Neurais Artificiais (RNAs) que se concentra em ajustar os pesos da rede de modo a maximizar a taxa de acerto das tendências dos preços de determinado ativo ao invés de minimizar o erro entre seus valores reais e previstos. Ao mesmo tempo, o método minimiza a probabilidade de overfitting, que é um problema muito frequente na aplicação de RNAs. Devido às características do problema encontradas em sua modelagem, como a presença de dois objetivos onde um deles apresenta uma função descontínua e não diferenciável, foi implementado um algoritmo evolucionário multiobjetivo para resolvê-lo. Este trabalho empregou dados reais de 10 ativos mais um índice da BM\&F Bovespa. Os experimentos computacionais realizados indicam que o modelo proposto, apesar de simples, apresentou resultados competitivos se comparados aos dos melhores trabalhos da área, que utilizam minimização de erro na sua implementação. Desta forma, a abordagem apresentada se mostrou bastante promissora, podendo contribuir com mais trabalhos dessa linha de pesquisa.
\end{abstract}

Keywords: Forecasting, financial series, artificial neural networks, multiobjective evolutionary algorithm.

Palavras-chaves: Predição, séries financeiras, redes neurais artificiais, algoritmo evolucionário multiobjetivo. 


\section{INTRODUÇÃO}

Recentemente as médias e grandes empresas têm aberto seu capital para o mercado financeiro, criando ações (ativos) negociáveis em bolsas de valores como Dow Jones, Nasdaq e BM\&F Bovespa. Um alto volume de negociação dessas ações geralmente implica em um aumento de seu preço de mercado e, consequentemente, em uma maior disponibilidade para investimentos.

O estudo do comportamento do mercado financeiro é relevante não só para estas empresas, mas também para todos os interessados em investir nelas. Uma tendência recente de interação com o mercado são os chamados robôs investidores, que são algoritmos em tempo real responsáveis por extrair dados, prever valores e efetuar transações de compra e venda. O foco deste trabalho está nos métodos de previsão, que constituem parte desses robôs.

O módulo de previsão do robô investidor é responsável por capturar os valores dos preços de um ou mais ativos, que receberam algum tipo de tratamento, e executar métodos que possam prever quais serão os preços em determinados instantes futuros, para que seja possível dizer se uma transação de compra ou de venda deve ser realizada. Se o valor do preço no dia seguinte a um determinado dia, escolhido para treinamento deste módulo do robô, aumentar, então vale a pena fazer uma compra do ativo em questão, por exemplo.

Os preços de ativos no mercado são disponibilizados para investidores apenas no formato de séries temporais por home brokers ${ }^{1}$ como o Metatrader, utilizado para coleta dos dados aplicados neste trabalho. Logo, devem ser aplicadas técnicas e modelos de previsão de séries temporais, como as Redes Neurais Artificiais (RNAs).

O treinamento dos dados de uma série temporal nada mais é do que um processo de otimização, visto que os algoritmos mais clássicos para esse tipo de problema, como o algoritmo Backpropagation (de P. Braga et al., 2007), buscam minimizar uma função de erro, que corresponde à diferença entre o valor real presente nos dados de treinamento e o valor previsto pela RNA.

Porém, o grande diferencial deste trabalho está em tentar predizer as tendências dos preços das séries financeiras, uma vez que esta característica é muito mais importante neste contexto do que a busca pelo menor erro.

A tendência determina a decisão do investidor em comprar ou vender determinado ativo, enquanto a minimização de erro tem como objetivo apenas a aproximação entre os valores real e previsto do preço em determinado dia, por exemplo, o que pode gerar informações equivocadas de tendência. Desta forma, o investidor pode tomar uma decisão que o prejudique financeiramente.

As principais diferenças entre os métodos de predição de séries financeiras empregados na literatura e os propostos neste trabalho estão retratadas abaixo:

\footnotetext{
1 Home brokers são softwares que realizam a intermediação entre a corretora da bolsa de valores e o investidor.
}

(1) A abordagem utilizada tem como um dos objetivos uma métrica responsável por contar a porcentagem de acertos de tendência. Desta forma, o algoritmo de treinamento prevê os preços de maneira a acertar as tendências dos mesmos o máximo possível, no período especificado.

(2) A abordagem deste trabalho propõe um mecanismo de tomada de decisão para auxiliar na escolha da melhor solução dentre as retornadas pelo algoritmo de treinamento, devido à característica multiobjetivo do problema.

(3) Finalmente, a abordagem proposta tem como seu segundo objetivo diminuir a probabilidade de overfitting, que é um problema muito comum quando utilizase redes neurais.

Realizou-se uma análise da distribuição estatística dos resultados da abordagem proposta, que foi comparada aos resultados de taxa de acerto obtidos por Nametala (2017). Este autor empregou diferentes versões do algoritmo Backpropagation na sua implementação, sendo este, um algoritmo de minimização de erro muito utilizado em RNAs.

Esta comparação indicou que os resultados alcançados neste trabalho foram promissores, uma vez que as taxas de acerto variaram entre $53 \%$ e $58 \%$.

Este artigo está organizado da seguinte forma: a Seção 2 consiste em uma análise da literatura do problema; a Seção 3 descreve a metodologia utilizada; a Seção 4 apresenta a proposta de solução discutida neste artigo; os resultados são apresentados e discutidos na Seção 5; por fim, o trabalho é encerrado na Seção 6 com as conclusões e propostas de continuidade.

\section{REVISÃO DE LITERATURA}

Nesta seção são discutidos alguns estudos que envolvem métodos computacionais para previsão de séries financeiras presentes na literatura que incluem a utilização de redes neurais artificiais.

Patel and Marwala (2006) usam redes neurais MLP e RBF (Radial Basis Function) para previsão de preços de fechamento dos índices Dow Jones, Johannesburg Stock Exchange All Share, Nasdaq 100 e Nikkei 225. O melhor e o pior resultado obtido quanto à taxa de acerto no movimento (alta/baixa) destes índices foram de $72 \%$ e $64 \%$, respectivamente.

Gambogi (2013) apresentou em um período de 5 anos para avaliação (2008 a 2012), um sistema de negociações que toma decisões de compra e venda no índice S\&P 500 por meio de redes MLP. Neste trabalho, o autor testou diversas configurações de redes neurais e escolheu as melhores baseado nos conjuntos de treinamento. As que apresentaram taxas de erro acima de $60 \%$ foram aplicadas ao período de operação. O sistema mostrou resultados superiores à evolução do próprio índice no período e, também, à técnica Buy and Hold.

Nametala (2017) utilizou um método híbrido combinando redes neurais MLP com preditores econométricos em um robô investidor de forma a predizer preços de fechamento de ativos da BM\&F Bovespa. Antes da previsão foi feito 
todo um tratamento dos dados, e os resultados desse método preditor foram usados para realizar negociações simuladas na bolsa brasileira, para que fosse avaliado seu desempenho em termos financeiros. A menor e a maior taxa de acerto obtida pelo robô implementado foram $53,03 \%$ e $61,4 \%$, respectivamente. Na média, a taxa de acerto foi $58,3 \%$.

É importante destacar que todos os trabalhos apresentados nesta seção, e muitos outros na literatura, fazem uso de alguma versão do algoritmo Backpropagation e/ou de algum outro método baseado em minimização de erro para treinamento de suas redes neurais.

O trabalho corrente é baseado em Nametala (2017) no que diz respeito à escolha dos ativos e ao tratamento feito com seus dados. No entanto, este apresenta um modelo mais simples.

\section{METODOLOGIA}

Os procedimentos e dados adotados para resolução do problema de previsão de séries financeiras estão descritos nesta seção.

\subsection{Dados de entrada do preditor}

Os preços de abertura, fechamento, mínima, máxima e o volume são os principais elementos das séries financeiras e são fundamentais para determinar uma cotação. Logo, podem conter informações suficientes para previsão dos preços de fechamento em instantes futuros, sendo estes, os valores previstos em todas as técnicas implementadas. Por isso, todos esses cinco elementos foram utilizados neste artigo como entrada para a rede neural.

Dessas cinco dimensões, quatro são utilizadas para compor uma das ferramentas de análises financeiras mais antigas e conhecidas, o candle. Um candle é um componente da série temporal presente no chamado "gráfico de velas" e tem este nome devido ao seu formato gráfico. Na Figura 1 é possível perceber claramente a presença dos preços citados anteriormente. É importante ressaltar que também podese incluir o volume, onde este se situa de forma alinhada e abaixo do candle gerado no mesmo pregão.

Existem dois tipos de volume, o de transações e o financeiro. O primeiro, corresponde ao conceito utilizado neste trabalho (sempre que for citada a palavra "volume", este deve significar "volume de transações") e trata da quantidade de negócios realizados em um determinado dia. Este valor é também chamado de "volume de ticks". O último, representa o valor total em moeda local negociado durante o dia.

\subsection{Topologia da rede neural}

A RNA utilizada foi uma rede feedforward, MLP, com cinco entradas (dimensões do candle mais o volume), uma camada escondida contendo 15 neurônios com função de ativação Sigmoide Logarítmica, e uma camada de saída com apenas um neurônio com função de ativação linear.

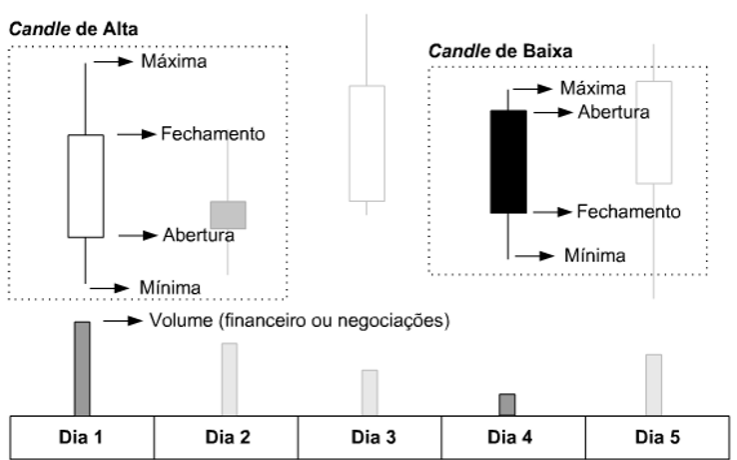

Figura 1. Componentes de um gráfico de candles

\subsection{Dados}

Os dados empregados neste trabalho para as previsões correspondem aos preços de mínima, abertura, máxima, fechamento e o volume de 10 ativos mais o índice Bovespa (BOVA 11) da bolsa de valores brasileira BM\&F Bovespa. Esses dados estão contidos no período entre 02/01/2009 e 30/12/2013. Os ativos escolhidos são listados abaixo:

- BBAS3 (Banco do Brasil)

- BBDC4 (Banco Bradesco)

- BVMF3 (Brasil Bolsa Balcão)

- CSNA3 (Companhia Siderúrgica Nacional)

- CYRE3 (Cyrela Brazil Realty)

- GGBR4 (Gerdau)

- ITSA4 (Itausa - Investimentos Itaú)

- PETR4 (Petrobras)

- USIM5 (Usiminas)

- VALE5 (Vale)

\subsection{Janelamento móvel}

O processo de previsão foi realizado através do chamado janelamento móvel. Esta abordagem é implementada em diversos estudos da área, como em Gambogi (2013) e Kaastra and Boyd (1996).

Através da Figura 2 é possível entender o processo da maneira que foi aplicado neste trabalho. O passo $j$ tem valor igual a $10 \mathrm{em}$ todos os métodos propostos. São realizadas 100 previsões em cada execução dos mesmos, portanto tem-se $k=10$ deslocamentos. O tamanho das janelas de todos os métodos é $n=30$, exceto o do método $M_{2}$, como será explicado posteriormente. A posição de partida da técnica de janelamento é escolhida aleatoriamente (seguindo uma distribuição uniforme) a cada execução. Esta posição deve estar contida no período correspondente aos dados utilizados.

Primeiramente, o algoritmo de previsão captura os dados de um determinado ativo, dentre os citados na Seção 3.3, iniciando assim, o processo de janelamento.

Os preços e o volume (tratados a priori) da janela de tamanho $n$ atual, como mostra a Figura 2, são normalizados e enviados para o treinamento da rede neural. Após isso, a rede prevê $j$ preços de fechamento $(p f(n+1)$ a $p f(n+j))$.

Com todas as previsões daquela iteração feitas, cada método implementado faz uma desnormalização e conversão para os valores absolutos dos preços de fechamento. 


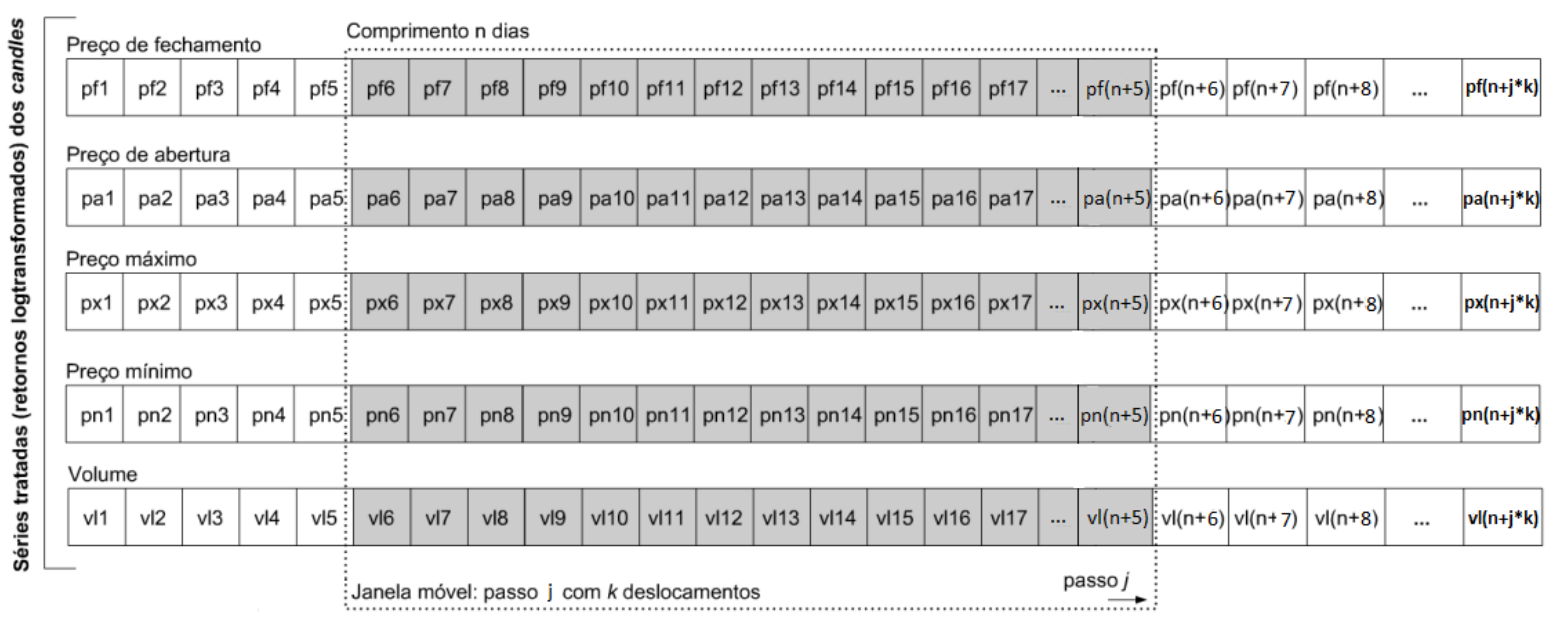

Figura 2. Janelamento móvel

Com o objetivo de obter os dados para realização do experimento de comparação dos métodos, os $j$ valores absolutos são avaliados pela métrica POCID (aplicada nesta pesquisa segundo o trabalho de Azevedo (2014)) e armazenados a cada iteração. A janela desliza com passo $j$ até concluir o número total de previsões daquela execução.

Ao final de todas as iterações desse algoritmo de previsão, é calculada a média dos $k$ valores de POCID armazenados anteriormente. Essa média foi considerada como o valor total de POCID de uma execução do algoritmo de previsão.

Esse tipo de abordagem foi selecionada para que se pudesse entender melhor a distribuição dos dados no processo de comparação.

\subsection{Normalização e tratamento dos dados}

Buscando diminuir ruídos das entradas da rede neural e minimizar o tempo de processamento, foram aplicadas neste trabalho uma forma de normalização e uma técnica de tratamento prévio dos dados utilizada por Miranda et al. (2012), onde esses autores transformaram dados de séries financeiras em retornos ${ }^{2}$ e na sequência aplicaram aos mesmos uma transformação logarítmica.

A técnica de normalização empregada trata-se de uma transformação linear dos dados para o intervalo $[0,1]$. Os preços tratados de acordo com o método explicado no parágrafo anterior (seus valores foram armazenados a priori para uso dos algoritmos) são normalizados em conjunto, por estarem todos na mesma escala. Na sequência, é normalizado o volume, uma vez que este se encontra em uma escala muito diferente. A normalização descrita está formulada em (1).

O conjunto de dados é então montado concatenando todas as dimensões. Por fim, os dados tratados e normalizados são inseridos como entrada da rede neural, começando o seu treinamento.

\footnotetext{
2 A tomada das primeiras diferenças entre os valores de preços de uma série é chamada em finanças de retornos.
}

$$
y_{i}=\frac{s_{i}-s_{\min }}{s_{\max }-s_{\min }}
$$

onde $s$ é uma série de preços, $i$ é o índice do preço na série, $s_{\min }$ é o menor valor de preço presente no conjunto e $s_{\max }$ é o maior valor. O mesmo se aplica se $s$ for uma série de volume.

Os cálculos de desnormalização e conversão para os valores absolutos dos preços de fechamento previstos, citados na subseção anterior, foram realizados a partir de (2) e (3), onde $p f_{\text {anterior }}$ é o último preço de fechamento conhecido (Nametala, 2017).

$$
\begin{aligned}
& s_{i}=y\left(s_{\max }-s_{\min }\right)+s_{\min } \\
& p f_{\text {previsto }}=e^{s_{i}}+p f_{\text {anterior }}
\end{aligned}
$$

\section{PROPOSTA DE SOLUÇÃO}

\subsection{Algoritmo proposto e representação da solução}

Os algoritmos mais populares para treinamento de RNAs do tipo do presente trabalho são os algoritmos Backpropagation (incluindo o que usa o algoritmo de LevenbergMarquardt). Porém a proposta deste artigo está em trocar este algoritmo de treinamento pelo Differential Evolution for Multiobjective Optimization (DEMO), uma vez que este tipo de algoritmo tem uma vantagem muito clara para problemas de otimização biobjetivo (Bandyopadhyay and Mukherjee, 2015).

Os objetivos escolhidos para este problema foram a métrica Percentage of Change in Direction (POCID) e a norma do vetor de pesos da rede, sendo que este vetor representa cada indivíduo da população do DEMO. A Equação (4) mostra a formulação do POCID:

$$
\begin{gathered}
\text { POCID }=\frac{1}{N} \sum_{i=1}^{N} a_{i}, \text { onde } \\
\left\{\begin{array}{l}
a_{i}=1, \text { se }\left(y_{i}-y_{i-1}\right)\left(r_{i}-r_{i-1}\right)>0 \\
a_{i}=0, \text { se }\left(y_{i}-y_{i-1}\right)\left(r_{i}-r_{i-1}\right) \leq 0
\end{array}\right.
\end{gathered}
$$


onde $y_{i}$ diz respeito ao valor previsto e $r_{i}$ ao valor real dos preços de fechamento presentes no conjunto de dados.

$O$ valor de POCID varia entre 0 e 1 , sendo que quanto mais próximo de 1 , maior o número de acertos das tendências dos preços de fechamento previstos em relação às dos preços reais. Se multiplicado por 100, o POCID nada mais é do que a taxa de acerto dessas tendências.

O modo como foi implementado cada indivíduo da população do algoritmo DEMO se encontra em (5):

$$
x=\left[\begin{array}{c}
w_{1, i, n} \\
w_{2, n}
\end{array}\right],
$$

onde o primeiro índice é o número da camada (1 é a camada escondida e 2 a de saída), $i$ corresponde ao número de entradas e varia de 1 a 5 , enquanto $n$ representa cada neurônio da camada escondida da RNA. Portanto, este índice varia de 1 a 15 . Com esta definição, é possível concluir que cada indivíduo $x$ tem dimensão $90 \times 1$.

O tamanho da população escolhido para o algoritmo foi 50, uma vez que este foi o valor mais alto encontrado de maneira empírica neste trabalho que gerou um tempo de execução aceitável do algoritmo de treinamento para obtenção dos resultados; o critério de parada corresponde ao número máximo de gerações, definido como 300. A probabilidade de recombinação e o fator de escala selecionados foram 0.8 e 0.9 , respectivamente.

Um dos desafios na implementação do algoritmo de treinamento consistiu na inicialização da população inicial de pesos. Logo, optou-se por utilizar o algoritmo de NguyenWidrow Nguyen and Widrow (1990), devido à sua eficiência e velocidade de execução em testes utilizando o Backpropagation com algoritmo de Levenberg-Marquardt para treinamento da rede de mesma arquitetura da aplicada neste trabalho.

Após o treinamento, o DEMO retorna um conjunto de vetores de pesos correspondentes à fronteira Pareto estimada. Na sequência, um deles é posteriormente inserido na rede neural para que seja feita a previsão dos preços de fechamento dos 10 dias seguintes. O método implementado para escolha do melhor vetor de pesos a ser utilizado no processo de previsão é descrito a seguir.

\subsection{Programação de compromissos (PC)}

Esta é uma técnica de análise de decisão multicritério, que visa indicar a solução de compromisso mais apropriada para um problema de otimização com mais de um objetivo. No método é identificada a solução, dentre as presentes na fronteira Pareto estimada, que está mais perto da solução utópica, ou ideal, mediante o uso de uma medida de proximidade (Lee and $\mathrm{Li}, 1993$ ).

A solução utópica minimiza (no contexto de um problema de minimização) todos os valores de função objetivo simultaneamente, e pode ser representada em (6), no caso de dois objetivos.

$$
\mathbf{f}^{*}=\left[f_{1}^{*}, f_{2}^{*}\right]
$$

Para estimar a melhor solução de compromisso, deve-se calcular o desvio de cada ponto da curva Pareto aproximada em relação à solução ideal, considerando também, a anti-ideal ou anti-utópica, para normalização dos objetivos caso estejam em escalas muito diferentes. A formulação do desvio se encontra em (7):

$$
d_{j}=\left[\sum_{i=1}^{m} w_{i} \frac{\left|f_{i}\left(x_{j}\right)-f_{i}^{*}\right|^{r}}{\tilde{f}_{i}-f_{i}^{*}}\right]^{1 / r},
$$

onde $f_{i}^{*}$ é a solução utópica para o objetivo $i, \tilde{f}_{i}$ é a solução anti-utópica para o objetivo $i, f_{i}\left(x_{j}\right)$ é o valor de função correspondente a cada solução $j$ da fronteira Pareto estimada (espaço de objetivos), $r$ é o valor da norma, no caso deste trabalho seu valor é $2, w_{i}$ é o valor dos pesos de cada objetivo, definidos como 0.6 no caso do primeiro (POCID), e 0.4 quanto ao segundo objetivo (norma dos pesos), $m$ é o número de objetivos, no caso deste trabalho $m=2$ e $d_{j}$ é o desvio daquela solução $j$ em relação à solução utópica.

A melhor solução de compromisso é aquela que apresentar o menor desvio. Buscando aprimorar o processo de tomada de decisão, foi evitada a escolha de pontos nos extremos da fronteira, mesmo que apresentassem o menor desvio, visto que eles nada mais são do que estimativas das soluções do problema de otimização de cada objetivo de forma independente. Portanto, sempre que isso ocorria, escolhiase o ponto com o segundo menor desvio.

Finalmente, este método foi definido neste artigo como PC de maneira a facilitar sua identificação na análise dos resultados.

\section{RESULTADOS}

Os resultados foram coletados através de 112 execuções do algoritmo de previsão para cada ativo, totalizando 1232 execuções. Este número de execuções foi escolhido de maneira a gerar um tamanho amostral grande, tornando a análise pela distribuição estatística dos resultados mais confiável.

Todos os valores de POCID gerados para todos os ativos pelo algoritmo proposto foram transformados no gráfico Boxplot da Figura 3.

O gráfico desta figura mostra que a distribuição dos resultados é aproximadamente simétrica em relação a sua mediana. Nota-se que os outliers não alteram a simetria da distribuição, pois há uma mesma quantidade desses pontos em cada extremo da mesma. O alto tamanho amostral juntamente com esta informação indica que a média da população de possíveis resultados para este método é próxima de $55 \%$. Além disso, grande parte dos valores de taxa de acerto resultantes do método podem variar em torno de $53 \%$ e $58 \%$.

Tomou-se como base os resultados apresentados em Nametala (2017), de maneira a avaliar de forma aproximada os retornos financeiros gerados pelo método apresentado neste artigo, uma vez que o trabalho discutido por esse autor utiliza um modelo com redes neurais muito parecido, porém muito mais robusto. Além disso, os ativos utilizados, os métodos de janelamento móvel e tratamento 


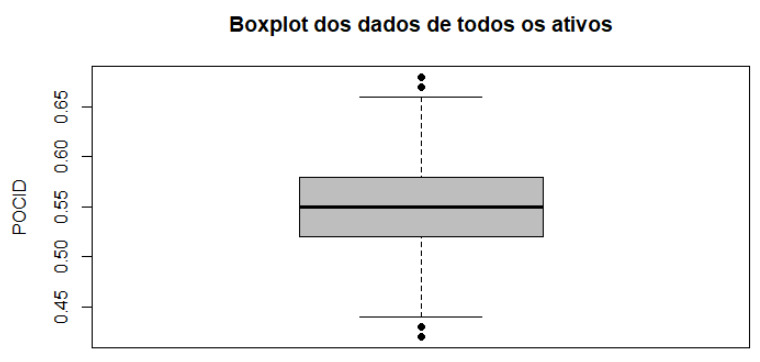

$\mathrm{DEMO}+\mathrm{PC}$

Figura 3. Boxplot dos dados de todos os ativos

de dados implementados, incluindo o período dos preços e do volume utilizados como entrada, são idênticos aos empregados no presente trabalho.

Esse autor implementa um Robô Investidor, no qual apresenta três módulos: (1) Extrator, que contém um método inteligente para seleção de ativos e tratamento dos dados de entrada do preditor; (2) Preditor, que apresenta um modelo híbrido com vários tipos de RNAs (sendo que estas usam o Backpropagation com algoritmo de LevenbergMarquardt, apresentado em Hagan and Menhaj (1994), como algoritmo de treinamento baseado em minimização de erro) e preditores econométricos para predição de preços; e (3) Negociador, que implementa algoritmos para manipulação de ordens de compra ou venda, gera relatórios para análise de desempenho e fornece medidas importantes ao módulo Preditor para que este se aprimore conforme o robô se desloca na série financeira.

O Robô Investidor apresentou uma média de 58,3\% na taxa de acerto e um retorno total líquido considerando toda a carteira de investimentos igual a $\mathrm{R} \$ 71.501,07$ no período de operação considerado. Desta forma, nota-se que o método implementado neste artigo é bastante promissor, uma vez que o modelo aplicado para o problema é bem mais simples e ainda assim apresentou resultados similares quanto à taxa de acerto.

\section{CONCLUSÕES}

O comportamento do mercado financeiro é algo muito complexo, pois os bens que são negociados nesse ambiente podem sofrer as mais diversas influências. Desta forma, qualquer contribuição que ajude a entender melhor os padrões que podem existir nesse tipo de problema é válida.

Este trabalho propôs um método de predição baseado em maximização dos acertos de tendência dos preços de fechamento de ativos em séries financeiras e que, ao mesmo tempo, visa a minimização da probabilidade de overfitting. O algoritmo evolucionário multiobjetivo DEMO implementado foi capaz de resolver o problema satisfatoriamente, gerando valores de taxa de acerto competitivos com os melhores trabalhos na área, o que consequentemente, pode gerar lucros significativos para um investidor.

Como proposta de continuidade, sugere-se a implementação de um modelo mais robusto quanto à extração e seleção dos dados, previsão e negociação, incluindo novas estratégias de tomada de decisão, de maneira a explorar melhor as soluções obtidas pelo DEMO.

Algo interessante a ser explorado seria a realização de análises e comparações mais sofisticadas do método proposto com técnicas de minimização de erro, utilizando modelos estatísticos e inferência, por exemplo.

Finalmente, como o presente trabalho visa propor um método para predizer melhor o comportamento de séries financeiras, faz sentido em um trabalho futuro, efetuar uma validação financeira do modelo, isto é, simular operações na $\mathrm{BM} \& \mathrm{~F}$ Bovespa e contabilizar os retornos gerados pela solução, comparando-os com outros trabalhos relacionados.

\section{AGRADECIMENTOS}

Este trabalho recebeu apoio da PRPq/UFMG e das seguintes agências de fomento brasileiras: FAPEMIG, CAPES e CNPq (404988/2016-4).

\section{REFERÊNCIAS}

Azevedo, C.R.B. (2014). Anticipation in Multiple Criteria Decision-Making Under Uncertainty. Ph.D. thesis, Universidade Estadual de Campinas.

Bandyopadhyay, S. and Mukherjee, A. (2015). An algorithm for many-objective optimization with reduced objective computations: A study in differential evolution. IEEE Transactions on Evolutionary Computation.

de P. Braga, A., Carvalho, A.P.L.F., and Ludernir, T.B. (2007). Redes Neurais Artificiais. LTC Editora, Rio de Janeiro.

Gambogi, J.A. (2013). Aplicação de Redes Neurais na Tomada de Decisão no Mercado de Ações. Master's thesis, Universidade de São Paulo.

Hagan, M.T. and Menhaj, M.B. (1994). Training feedforward networks with the marquardt algorithm. IEEE Transactions on Neural Networks, 5(6), 989-993. doi: 10.1109/72.329697.

Kaastra, I. and Boyd, M.S. (1996). Designing a neural network for forecasting financial and economic time series. Neurocomputing, 10, 215-236.

Lee, E.S. and Li, R. (1993). Fuzzy multiple objective programming and compromise programming with pareto optimum. Fuzzy Sets and Systems, 53(3), 275 - 288.

Miranda, A., Ceretta, P.S., and Lopes, L.F.D. (2012). Estratégias de mercado acionário utilizando previsão de redes neurais em comparação com modelos autorregressivos. Revista da Administração, 8(1), 42-59.

Nametala, C.A.L. (2017). Construção de um Robô Investidor baseado em Redes Neurais Artificiais e Preditores Econométricos. Master's thesis, Universidade Federal de Minas Gerais.

Nguyen, D. and Widrow, B. (1990). Improving the learning speed of 2-layer neural networks by choosing initial values of the adaptive weights. Proceedings of the International Joint Conference on Neural Networks, 3, 21-26.

Patel, P.B. and Marwala, T. (2006). Forecasting closing price indices using neural networks. IEEE Conference on Systems, Man and Cybernetics. 